\title{
Isolation of Pathogenic Bacteria and Their Antibiotic Sensitivity Profiles in Hospitalized Febrile Neutropenic Children with Acute Lymphoblastic Leukaemia
}

\author{
Yeamin $\mathrm{MB}^{1}$, Khatun $\mathrm{MN}^{2}$, Mollah $\mathrm{LR}^{3}$, Rahman $\mathrm{R}^{4}$, Ahamed $\mathrm{MT}^{5}$, Mir IUMAK ${ }^{6}$, Siddique $\mathrm{R}^{7}$, \\ Akter $\mathrm{M}^{8}$, Yasmin $\mathrm{F}^{9}$, Begum $\mathrm{F}^{10}$, Jamal $\mathrm{CY}^{11}$
}

\begin{abstract}
Background: Acute lymphoblastic leukemia (ALL) is the commonest malignancies in childhood. Common obstacle in the treatment of ALL is febrile neutropenia and its complications. Objectives: To identify bacteria causing infection, their isolation rate and antibacterial sensitivity pattern in hospitalized febrile neutropenic children with ALL in different cycle of chemotherapy. Methodology: This observational study conducted in 2014 - 2015 in the department of paediatric haematology and oncology, Bangabandhu Sheikh Mujib Medical University (BSMMU), Dhaka Bangladesh. Sixty febrile neutropenic episodes from 52 diagnosed cases of ALL aged 0 tol 8 years were included. Complete blood count, blood culture, urine microscopy and culture, serum alanine aminotransferase, serum creatinine were done in every patient. X-ray chest, stool microscopy and culture, pus, wound, throat and aural swab for culture \& sensitivity were done in selective patient. Results: Bacterial infection was confirmed by culture in 15 (25\%) episodes from 60 febrile neutropenic episodes. Fifteen (25\%) organisms were isolated from the study subjects from sample of blood (60\%), pus (13.3\%), aural swab (13.3\%), wound swab (6.7\%) and throat swab $(6.7 \%)$ respectively. All isolates were gram negative. The organism isolated were Klebsiella spp. 5 (33.31\%), E. coli 4 (26.7\%), Acinetobacter 3 (20\%), Pseudomonas $2(13.3 \%)$ and only one (6.7\%) Enterobacter species. All the isolates of the Klebsiella spp., E. coli and Acinetobacter spp. were resistant to amoxicillin. All isolated E. coli were resistant to cotrimoxazole, ceftazidime, ceftriaxone, cefotaxime and ciprofloxacin, Acinetobacter spp. Isolated were 100\% sensitive to imipenem, colistin sulphate \& piperacillin-tazobactam and resistant to cotrimoxazole and cephradine. All Pseudomonas spp. showed 100\% sensitivity to imipenem, amikacin, ciprofloxacin \& colistin and resistances to ceftazidime. Conclusion: The species of Klebsiella were the predominant causative bacterial agent followed by Escherichia coli, Acinetobacter spp, pseudomonas spp. and Enterobacter spp. They showed resistance to commonly prescribed antibiotics ceftazidime, gentamicin, ceftriaxone \& ciprofloxacin and sensitive to imipenem, colistin-sulphate \& piperacillin-tazobactam.
\end{abstract}

Keywords: Neutropenia, Pathogenic Bacteria, Culture and sensitivity.

\section{Author's}

1. *Md. Bani Yeamin, Department of Pediatric Hematology and Oncology, Chattogram Medical College, Chattogram, Bangladesh.

2. Mst. Nazma Khatun, Department of Microbiology, Shaheed Tajuddin Ahmed Medical College Hospital, Gazipur, Bangladesh.

3. Lutfor Rahman Mollah, Department of Pediatric Hematology and Oncology, Sir Salimullah Medical College, Dhaka, Bangladesh.

4. Rezanur Rahman, Department of Pediatric Hematology and Oncology, Dhaka Medical College, Dhaka, Bangladesh.

5. Mohammad Tanvir Ahamed, Department of Pediatric Hematology and Oncology, Dhaka Medical College, Dhaka, Bangladesh.

6. I U M A Kawsar Mir, Department of Pediatrics, Shaheed Tajuddin Ahmed Medical College Hospital, Gazipur, Bangladesh.

Page | 30
7. Rasel Siddique, Department of Pediatric Hematology and Oncology, Bangabandhu Sheikh Mujib Medical University, Dhaka, Bangladesh.

8. Mehnaz Akter, Department of Pediatric Hematology and Oncology, Dhaka Medical College, Dhaka, Bangladesh.

9. Farida Yasmin, Department of Paediatric Haematology and Oncology, National Institute of Cancer Research and Hospital, Mohakhali, Dhaka, Bangladesh.

10. Ferdousi Begum, Department of Paediatric Haematology and Oncology, National Institute of Cancer Research and Hospital, Mohakhali, Dhaka, Bangladesh.

11. Chowdhury Yakub Jamal, Department of Pediatric Hematology and Oncology, Bangabandhu Sheikh Mujib Medical University, Dhaka, Bangladesh.

Correspondence: Md. Bani Yeamin, Department of Pediatric Hematology and Oncology, Chattogram Medical College, Chattogram,Bangladesh, E-mail: dr.baniyeamin@gmail.com, Contact: +880 1785-901909.

Haematol J Bangladesh 2019; 3(2):30-34 


\section{Original Article}

\section{Introduction}

Acute leukemia is one of the most common childhood malignancies. Childhood acute lymphoblastic leukaemia (ALL) is also common in our country. Cancer treatment is associated with infections, in spite of the progress made in the development of antibiotics, is still a major concern for healthcare professionals. On the other hand, assessment of the type of infection presented after chemotherapy plays a major role in achieving a better treatment outcome1. A correct prediction of the type of infection at the immediate onset of fever before culture results is available. On the contrary, late decision-making and wrong predictions can not only lead to the spread of infection, but can also result in failure of chemotherapy, delayed completion of remission, and poor treatment outcome. Febrile neutropenia is the most frequent manifestation of a potentially lethal complication of current intensive chemotherapy regimens. And it is also oncology related medical emergency 2,3 . The pathogens isolated from culture in neutropenic fever have changed over time and may be location dependent.

Culture isolates from febrile neutropenic patients were once predominantly gram negative-organisms ${ }^{4}$. There were major changes in the type and range of pathogens causing infection in neutropenic patients during the last decades 5 . Clinicians should be aware of the predominant pathogens and an antibiogram that depicts the in vitro susceptibility patterns of the most prevalent pathogens in their own institution to select an efficient initial empirical therapy 6 . So, with this view, aim of our study is to assess the frequency of bacterial infection and their antibacterial sensitivity profiles in hospitalized febrile neutropenic children with acute lymphoblastic leukemia in different cycle of chemotherapy.

\section{Methodology}

This was a cross sectional observational study conducted from November 2014 to November 2015 in the department of paediatric haematology and oncology, Bangabandhu Sheikh Mujib Medical University (BSMMU). Sixty febrile neutropenic episodes from 52 diagnosed cases of ALL with febrile neutropenic children aged 0 to18 years were included during one-year study period after fulfilling the criteria of case definition. Informed consent from respective parents and legal guardian obtained prior to inclusion Page | 31 of any child. History was taken for any symptom of sepsis. Then proper physical examination carried out. Complete blood count (CBC), blood culture and sensitivity, urine routine microscopic examination and culture \& sensitivity, serum glutamic pyruvic transaminase, serum creatinine were done in every patient. X-ray chest, stool routine microscopic examination and culture \& sensitivity, pus, wound, throat and aural swab for culture \& sensitivity were done in selected patient according to sites of infection in the respective department of BSMMU.

\section{Study procedure}

Their full initial presentations, initial total count, history of recent chemotherapy with their intensity, history of any associated co morbidity like diarrhea, shock, DIC, severe bleeding manifestation \& full physical examination were recorded. Complete blood count (CBC) was done by Sysmax ${ }^{\circledR}$ automated analyzer, serum glutamic pyruvic transaminase, and serum creatinine were done in every patient in the department of Paediatric Haematology and Oncology. $\mathrm{X}$-ray chest was done in the radiology department, BSMMU and on emergency cases bed side X-ray was done in selective patients. All aseptic precautions were maintained carefully before and after specimen collection. Blood culture and sensitivity (Automated BACTEC 9240 machine), urine routine microscopic examination \& culture and sensitivity were done in every patients and stool routine microscopic examination \& culture and sensitivity, pus, wound, throat \& aural swab for culture and sensitivity were done in selected patient according to sites of infection in the department of microbiology, BSMMU. Venous blood was collected for complete blood count, metabolic workup and blood culture.

\section{Statistical analysis of data}

After collection, data editing, compilation and analysis was done manualy. Statistical analysis was done both manually and window-based software devised with Statistical Packages for the Social Sciences (SPSS).

\section{Ethical implication}

Prior to the commencement of this study, the thesis protocol was approved by the Institutional Review Board, BSMMU, Dhaka. The thesis protocol was explained to parents or legal guardians about my study

Haematol J Bangladesh 2019; 3(2):30-34 
before taking the consent. Then a written consent was taken from each patient's legal guardian. Every precaution was taken so that study will not cause any harm or delay the treatment of cases. They had liberty to exclude their child from the study at any time.

\section{Results}

Total 60 febrile neutropenic episodes from 52 acute lymphoblastic leukaemic children were studied, 35 males and 17 females, with a mean age 5.5 years. (Fig 1, Table 1) Primary site of infection could not be suspected clinically in $19(31.6 \%)$ episodes. Bacterial infection was confirmed by culture in 15 (25\%) episodes from 60 febrile neutropenic episodes.

Table 1: Distribution of febrile neutropenic episodes with ALL patients according to age $(n=60)$.

\begin{tabular}{lc}
\hline Age Groups (years) & $\mathbf{n}(\boldsymbol{\%})$ \\
\hline$\leq 2$ & $10(16.7)$ \\
$2.1-5.0$ & $27(45.0)$ \\
$5.1-10.0$ & $17(28.3)$ \\
$>10$ & $6(10.0)$ \\
Total & $60(100.0)$ \\
Mean \pm SD & $5.47 \pm 3.57$ \\
Range (Min-Max) & $0.5-16.0$ \\
\hline
\end{tabular}

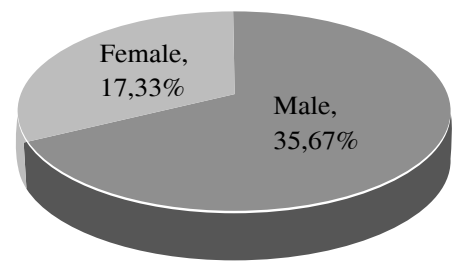

Figure 1: Sex distribution of the patients.

Seven patients were admitted in more than one episode and two of them were culture positive in more than one episode. Fifteen (25\%) organisms were isolated from the study subjects of which blood 9 $(60 \%)$, pus $2(13.3 \%)$, aural swab $1(13.3 \%)$, wound swab $1(6.7 \%)$ and throat swab $1(6.7 \%)$. All isolates were gram negative. (Fig. 2)

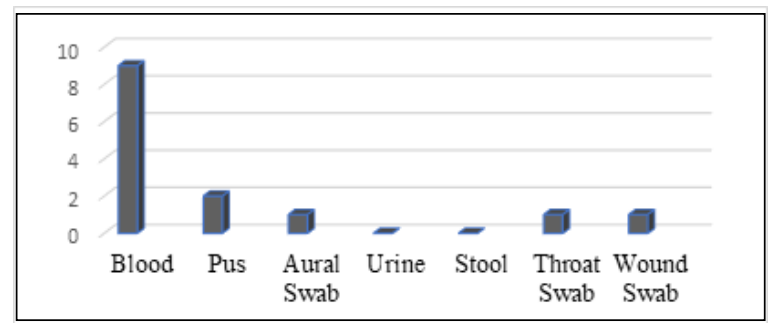

Figure 2: Result of culture positivity of different specimen of febrile neutropenic episodes.

Haematol J Bangladesh 2019; 3(2):30-34
The most common organism was Klebsiella spp 5 $(33.31 \%)$, followed by E. coli 4 (26.7\%), Acinetobacter $3(20 \%)$, Pseudomonas $2(13.3 \%)$ and only one $(6.7 \%)$ was Enterobacter species. Maximum isolation of bacteria $13(32.5 \%)$ of 40 episodes was in induction phase of chemotherapy. (Fig. 3)

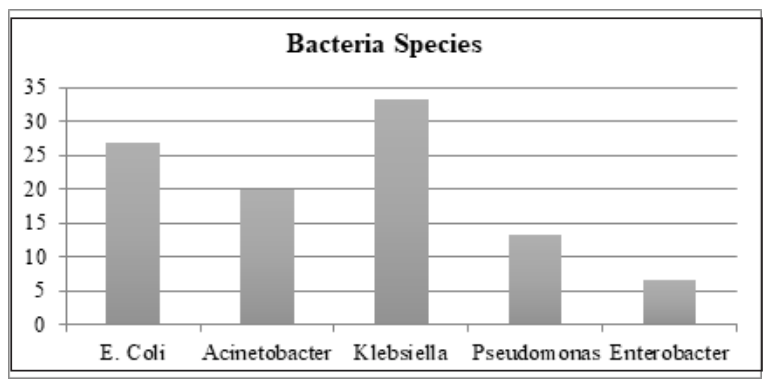

Figure 3: Isolation of Bacterial species from Culture

All the isolated of the Klebsiella spp., E. coli and Acinetobacter spp. were resistant to amoxicillin and Cephradine. Eighty (80\%) of Klebsiella spp. were resistant to cotrimoxazole, ceftazidime, ceftriaxone, cefotaxime and cefuroxime. All isolated the E. coli were resistant to cotrimoxazole, ceftazidime, ceftriaxone, cefotaxime and ciprofloxacin followed by $75 \%$ were resistant to amikacin. All isolated the Acinetobacter spp. $100 \%$ sensitive to imipenem, colistin sulphate \& piperacillin-tazobactam and resistant to cotrimoxazole and cephradine. All Enterobacter spp. showed 100\% sensitive of imipenem \& amikacin and resistant to other antibiotics used in the study. All Pseudomonas spp. showed $100 \%$ to imipenem, amikacin, ciprofloxacin $\&$ colistin and resistant to ceftazidime. (Table 2)

Table 2: Resistance pattern of antibiotics against commonly isolated pathogens (gram negative) of febrile neutropenic episodes with ALL.

\begin{tabular}{|c|c|c|c|c|c|}
\hline Antibiotics $\mathrm{E}$ & $\begin{array}{c}\text { Escherichia } \\
\text { Coli }\end{array}$ & $\begin{array}{c}\text { Acinetobacter } \\
\text { spp }\end{array}$ & $\begin{array}{c}\text { Klebsiella } \\
\text { spp }\end{array}$ & $\begin{array}{c}\text { Pseudomonas } \\
\text { spp }\end{array}$ & $\begin{array}{c}\text { Enterobacter } \\
\text { spp }\end{array}$ \\
\hline Amoxycilin & $4(100.0)$ & $3(100.0)$ & $5(100.0)$ & - & $1(100.0)$ \\
\hline Cotrimoxazole & le $4(100.0)$ & $3(100.0)$ & $4(80.0)$ & - & $1(100.0)$ \\
\hline Nalidixic & $4(100.0)$ & $2(66.7)$ & $3(60.0)$ & - & - \\
\hline Gentamicin & - & $1(33.3)$ & $2(66.7)$ & $1(50.0)$ & $1(100.0)$ \\
\hline Ceftazidime & $4(100.0)$ & $2(66.7)$ & $4(80.0)$ & $2(100.0)$ & $1(100.0)$ \\
\hline Amikacin & $3(75.0)$ & $1(33.3)$ & $2(40.0)$ & 0 & 0 \\
\hline Imipenem & $2(50.0)$ & 0 & $1(20.0)$ & 0 & 0 \\
\hline Cephradine & $2(100.0)$ & $3(100.0)$ & $4(100.0)$ & - & $1(100.0)$ \\
\hline Ciprofloxacin & n $4(100.0)$ & $1(33.3)$ & $1(20.0)$ & 0 & $1(100.0)$ \\
\hline Ceftriaxone & $4(100.0)$ & $2(66.7)$ & $4(80.0)$ & - & $1(100.0)$ \\
\hline Cefotaxime & $4(100.0)$ & $2(66.7)$ & $4(80.0)$ & - & $1(100.0)$ \\
\hline Cefuroxime & $4(100.0)$ & $2(66.7)$ & $4(80.0)$ & - & $1(100.0)$ \\
\hline Aztreonam & $4(100.0)$ & $2(66.7)$ & $4(80.0)$ & $1(50.0)$ & $1(100.0)$ \\
\hline Netilmicin & $2(50.0)$ & $1(33.3)$ & $1(20.0)$ & 0 & $1(100.0)$ \\
\hline Cefepime & - & - & & $1(50.0)$ & - \\
\hline Tazobactam & $2(50.0)$ & 0 & - & $1(50.0)$ & - \\
\hline Piperacillin & & & & & \\
\hline Ticarcillin & - & $2(66.7)$ & - & 0 & - \\
\hline Colistin & - & 0 & - & 0 & - \\
\hline
\end{tabular}


Original Article

\section{Discussion}

Infections in the immunocompromised host as a result of cancer chemotherapy is an important problem in the present day-to-day treatment care, as they are associated with an increased incidence of neutropenic infectious complication, which in turn influences the outcome of the chemotherapeutic response, and thereby morbidity and mortality, in these patients 7 .

One study discussed that blood was the most common site of infection and $85.4 \%$ of them had gram-negative bacteria and also stated that source of $56 \%$ of isolated organisms was blood and gram-negative bacteria accounted for $78 \%$ of isolated organisms from febrile neutropenic patients in hematological malignancies 8 .

The most common organisms were Escherichia coli (43\%) and Klebsiella pneumonia (17.4\%) and revealed among gram negative-organism E.coli (30.2\%) was followed by Klebsiella Pneumoniae $(20.9 \%) 9$. In this study, all $(100 \%)$ the isolated organisms were gram negative and $60 \%$ of these were from blood but there were no growth in stool and urine. The most common isolated organisms were Klebsiella spp. (33.3\%) followed by Esch. Coli (26.7\%) and Acinetobacter spp. (20.0\%). In this study, all the isolated of the Klebsiella spp., E. coli and Acinetobacter spp. were resistant to amoxicillin and Cephradine. Eighty (80\%) of Klebsiella spp. were resistant to cotrimoxazole, ceftazidime, ceftriaxone, cefotaxime and cefuroxime. All isolated the $E$. coli were resistant to cotrimoxazole, ceftazidime, ceftriaxone, cefotaxime and ciprofloxacin followed by $75 \%$ were resistant to amikacin. All isolated the Acinetobacter spp. showed $100 \%$ sensitive to imipenem, colistin sulphate \& piperacillin-tazobactam and resistant to cotrimoxazole and cephradine. All Enterobacter spp. showed $100 \%$ sensitive of imipenem \& amikacin and resistant to other antibiotics used in the study. All Pseudomonas spp. showed $100 \%$ sensitive to imipenem, amikacin, ciprofloxacin \& colistin and resistant to ceftazidime. Mahallawy et al. (2005) has reported very high resistance rates for gram negative pathogens, reaching $60 \%$ for ceftazidime, $50 \%$ for amikacin and imipenem and $40 \%$ for piperacillin-tazobactam, cefepime and ciprofloxacin10. Another popular study Aslan et al. (2012) has revealed the antimicrobial sensitivity patterns of gram-negative organisms to imipenem (89.2\%). Kwon et al. (2013) has also stated

Page | 33 that the resistance rate of gram-negative bacteria was amikacin $10.6 \%$, imipenem $4.4 \%$. In this study very high resistance rates were reported to gram negative bacteria11. The most probable explanation for these findings is the increased use of antibiotics in the hospital, as evidenced by a profound resistance to both amikacin and ceftazidime, which have been used extensively as first-line empirical therapy.

\section{Conclusion}

Febrile neutropenic patients with acute lymphoblastic leukaemia underwent chemotherapy were in a major risk for infection. The present study, $25 \%$ of febrile neutropenic episodes were bacterial culture positive in different phases of chemotherapy and all were gram-negative with klebsiella spp. as major isolate followed by Escherichia coli, Acinetobacter spp, pseudomonas spp. and enterobacter spp. Sixty (60\%) of these bacteria were isolated from blood and mostly $13(32.5 \%)$ during induction phase of chemotherapy. These bacteria were highly resistance to ceftazidime, gentamicin, ceftriaxone, ciprofloxacin, amikacin \& cefotaxime and sensitive to imipenem, colistin-sulphate \& piperacillin-tazobactam.

\section{References}

1. Ahmadzadeh A, Varnasseri M, Jalili MH, Maniavi F, Valizadeh A, Mahmoodian M, Keyhani M. Infection pattern of neutropenic patients in post-chemotherapy phase of acute leukemia treatment. Hematology reports. 2013 Dec;5(4).

2. Wicki S, Keisker A, Aebi C, Leibundgut K, Hirt A, Ammann RA. Risk prediction of fever in neutropenia in children with cancer: a step towards individually tailored supportive therapy? Pediatric blood \& cancer. 2008 Dec;51(6):778-783.

3. Collin BA, Leather HL, Wingard JR, Ramphal R. Evolution, incidence, and susceptibility of bacterial bloodstream isolates from 519 bone marrow transplant patients. Clinical infectious diseases. 2001 Oct 1;33(7):947-953.

4. Viscoli C, Varnier O, Machetti M. Infections in patients with febrile neutropenia: epidemiology, microbiology, and risk stratification. Clinical Infectious Diseases. $2005 \mathrm{Apr}$ 1;40(Supplement_4):S240-245.

5. Akova M. Emerging problem pathogens: A review of resistance patterns over time. International Journal of Infectious Diseases. 2006 Sep 1;10:S3-8.

6. Sipsas NV, Bodey GP, Kontoyiannis DP. Perspectives for the management of febrile neutropenic patients with cancer in the 21st century. Cancer: Interdisciplinary International Journal of the American Cancer Society. 2005 Mar 15;103(6): 1103-1113.

Haematol J Bangladesh 2019; 3(2):30-34 
7. Donowitz GR, Maki DG, Crnich CJ, Pappas PG, Rolston KV. Infections in the neutropenic patient-new views of an old problem. ASH Education Program Book. 2001 Jan 1;2001(1):113-139.

8. Karanwal, A.B., Parikh, B.J., Goswami, P., Panchal, H.P., Parekh, B.B. and Patel, K.B., 2013. Review of clinical profile and bacterial spectrum and sensitivity patterns of pathogens in febrile neutropenic patients in hematological malignancies: A retrospective analysis from a single center. Indian journal of medical and paediatric oncology: official journal of Indian Society of Medical \& Paediatric Oncology, 34(2), p.85.

9. Aslan S, Citak EC, Yis R, Degirmenci S, Arman D. Bacterial spectrum and antimicrobial susceptibility pattern of bloodstream infections in children with febrile neutropenia: experience of single center in southeast of Turkey. Indian journal of microbiology. 2012 Jun 1;52(2):203-208.
Original Article

10. El-Mahallawy H, Sidhom I, El-Din NA, Zamzam M, El-Lamie MM. Clinical and microbiologic determinants of serious bloodstream infections in Egyptian pediatric cancer patients: a one-year study. International journal of infectious diseases. 2005 Jan 1;9(1):43-51

11. Kwon JC, Kim SH, Choi JK, Cho SY, Park YJ, Park SH, Choi SM, Lee DG, Choi JH, Yoo JH. Epidemiology and clinical features of bloodstream infections in hematology wards: one-year experience at the catholic blood and marrow transplantation center. Infection \& chemotherapy. 2013 Mar 1;45(1):51-61. 